\title{
O Número de Manchas Solares, Índice da Atividade do Sol
}

Sunspot Number, Solar Activity Index

\author{
Ezequiel Echer ${ }^{(1)}$, Nivaor Rodolfo Rigozo ${ }^{(1,2)}$, Daniel Jean Roger Nordemann ${ }^{(1)}$, \\ Luiz Eduardo Antunes Vieira ${ }^{(1)}$, Alan Prestes ${ }^{(1)}$ e Heloisa Helena De Faria ${ }^{(1)}$ \\ ${ }^{1}$ Instituto Nacional de Pesquisas Espaciais - INPE, \\ POB 515, 12201-970 São José dos Campos, Brazil. \\ Fone: 55123456840 - Fax 55123456810. \\ ${ }^{2}$ Faculdade de Tecnologia Thereza Porto Marques - FAETEC, \\ CEP 12308-320, Jacareí, Brazil \\ E-mail:ezequiel@dge.inpe.br,rodolfo@dge.inpe.br,nordeman@dge.inpe.b,eduardo@dge.inpe.br, \\ alan@dge.inpe.br,eHeloisa@dge.inpe.br
}

Recebido em 17 de fevereiro, 2003. Aceito em 4 de abril, 2003.

\begin{abstract}
O número de manchas solares é o índice mais antigo da atividade solar, com observações de manchas solares estendendo-se desde 1611. Ele serve como uma medida da atividade magnética geral do Sol. Neste trabalho apresentamos uma descrição das características físicas das manchas solares, a definição do número de manchas solares, uma análise espectral do mesmo e uma reconstrução de 1000 anos.
\end{abstract}

\begin{abstract}
The sunspot number is the longest solar activity index available with observations since 1611 . It is a measure of the general state of solar magnetic activity. In this work we present a desciption of sunspot physical caracteristics, the definition of the sunspot number, a spectral analysis and a reconstruction for the last 1000 years of the sunspot number.
\end{abstract}

\section{Introdução}

O Sol é uma estrela ordinária da seqüência principal e tem idade estimada de 4,5 bilhões de anos. A distância média Terra-Sol (1 Unidade Astronômica) equivale a 150 milhões de km. Sua composição é de aproximadamente $90 \%$ de Hidrogênio $(\mathrm{H}), \sim 10 \%$ de Hélio $(\mathrm{He})$ e aproximadamente $0,1 \%$ de elementos mais pesados: Carbono (C), Nitrogênio $(\mathrm{N})$ e Oxigênio $(\mathrm{O})$. A temperatura superficial do Sol é de 5780K, mas no núcleo a temperatura chega a milhões de graus. Esta alta temperatura faz com que os gases sejam quase totalmente ionizados, formando um plasma.

Embora o Sol, em seu período calmo, seja de imenso interesse astronômico, por ser a única estrela cujas características espaciais são observáveis em detalhe com os telescópios de que dispomos, a maior parte dos efeitos geofísicos de interesse resulta do Sol ativo, exceto aqueles devido a irradiância solar.

As características mais claras da variabilidade solar são as mudanças com o tempo no número de manchas solares vistas na metade visível do Sol (Stuiver e Quay, 1980). Os registros observados do número de manchas solares mostram um ciclo regular médio da atividade solar próximo dos 11 anos. Não existe ainda uma explicação física completa para a observação do ciclo solar (Eddy, 1976). Modernas teorias atribuem as características periódicas das manchas solares à existência de um dínamo solar em que a convecção e a rotação diferencial da superfície interagem para amplificar e manter um assumido campo magnético inicial (Leighton, 1969). Modelos de dínamo são bem sucedidos na reprodução de certas características do ciclo de 11 anos, mas ainda não é possível explicar a variação de amplitude nas épocas de máximo e de outras mudanças características de longo período (Eddy, 1976).

\section{Características Físicas das Man- chas Solares}

Manchas solares são regiões mais frias (cerca de $2000 \mathrm{~K}$ ) e mais escuras (por efeito de contraste) do que a fotosfera solar circunvizinha. Elas emitem menos energia do que a fotosfera em geral porque possuem intensos campos magnéticos (cerca de 1000 vezes mais intensos que a superfície solar normal, $0,1 \mathrm{~T}$ contra $10^{-4} \mathrm{~T}$, onde $\mathrm{T}=\mathrm{Tesla}$ ) que parcialmente bloqueiam a energia transmitida para cima pelas células de convecção na região sub-fotosférica (Eddy, 1976; Schove, 1983; Kivelson and Russel, 1995). As manchas solares são geradas por campos magnéticos toroidais que são trazidos à superfície solar, por efeito de empuxo. No decorrer de cada ciclo solar de 11 anos esses tubos ascen- 
dem e irrompem na superfície solar, e as aberturas dos tubos de fluxo ou manchas, aparecem primeiro em médias latitudes heliográficas médias $\left(30^{\circ}-40^{\circ}\right)$ em ambos hemisférios. No máximo do ciclo solar elas estão concentradas na região entre $10^{\circ}-20^{\circ}$, próximo ao equador solar. As manchas solares têm sido observadas a olho nu pelo menos desde 1000 anos A. C. na antiga China (Eddy, 1976) e telescopicamente na Europa desde 1611 (Eddy, 1976; Schove, 1983). A Figura 1a apresenta uma fotografia na linha $\mathrm{H}$-alfa (filtro usado na linha de emissão intensa, na região visível do vermelho, em torno de $650 \mathrm{~nm}, 1 \mathrm{~nm}=10^{-9} \mathrm{~m}$ ) da superfície do Sol, onde as manchas solares aparecem como características mais escuras (www.sunspotcycle.com), e na Figura 1b temos uma visão mais detalhada de um grupo de manchas solares (http://www.solarviews.com).

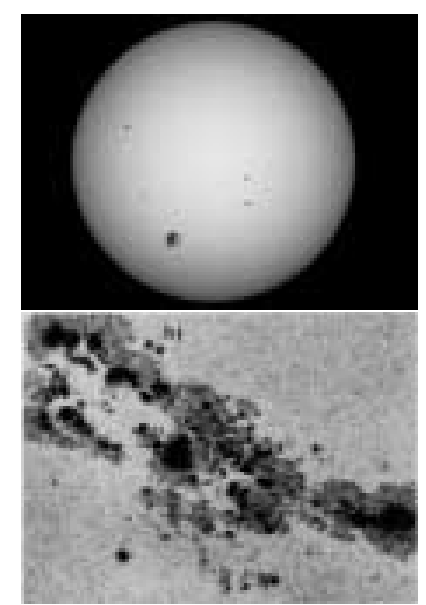

Figura 1. (a) Imagem da superfície solar na linha H-alpha, apresentando às características mais escuras, as manchas solares, e (b) visão mais detalhada de um grupo de manchas solares. Fonte: NASA, Sunsposts and Solar Cycle, http://www.sunspotcycle.com; http://www.solarviews.com/eng/sun.htm

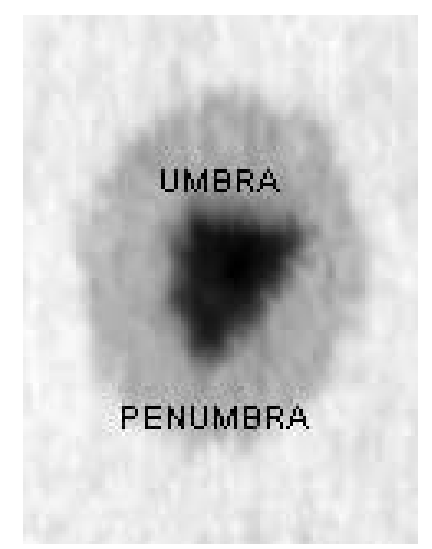

Figura 2. Imagem da superfície solar na linha H-alpha, apresentando as características das manchas solares, a parte interna mais escura (umbra) e a parte circundante, mais clara (penumbra). Fonte: NASA, Sunsposts and Solar Cycle, http://www.sunspotcycle.com

Uma mancha solar grande pode cobrir uma área de cerca de 700 vezes a área superficial da Terra, tendo $20 \times 10^{6} \mathrm{~m}$ em diâmetro (Hoyt e Schatten, 1997). A estrutura da mancha solar é apresentada na foto, em detalhe, da Figura 2. A região central, mais escura, é chamada umbra, a uma tempe- ratura de cerca de $4100 \mathrm{~K}$ e com campo magnético total de $0,3 \mathrm{~T}$, cercada por uma região fronteiriça acinzentada, a penumbra, de filamentos radiais claros e escuros. Penumbras têm em geral 2,5 vezes o diâmetro das umbras, que variam entre $300-2500 \mathrm{~km}$ em média, mas apenas $50 \%$ das manchas solares desenvolvem uma penumbra. O campo magnético é quase vertical na umbra, e mais horizontal nas penumbras.

A polaridade magnética de uma mancha pode ser norte ou sul. Manchas complexas podem apresentar uma mistura de ambas polaridades, o que pode ser compreendido como o resultado da fusão de manchas solares menores nãocomplexas. Entretanto, as manchas tendem geralmente, a ocorrer em pares de polaridade oposta, como apresentado na Figura 3. Um grupo de manchas é orientado primariamente na direção leste - oeste com relação a um observador fixo na Terra (ao meio-dia, a borda oeste do Sol estará mais próxima do horizonte oeste da Terra). A mancha mais a oeste de um grupo é conhecida como mancha líder ou precedente, e as manchas mais a leste como as seguidoras. A mancha líder é em geral a $1^{a}$ mancha a se formar e a última a desaparecer. Também é tipicamente a $1^{a}$ mancha a formar uma penumbra e a última a perdê-la, além de ser a maior mancha na vida de um grupo. Os grupos de manchas em um dado hemisfério solar tendem a ter líderes da mesma polaridade magnética; líderes no hemisfério norte geralmente têm a mesma polaridade que manchas seguidoras no hemisfério sul. Também em geral a mancha líder tem a mesma polaridade do campo magnético de "background" do hemisfério residente. Como a cada 11 anos a polaridade magnética solar se inverte, manchas líderes em um dado hemisfério terão polaridade oposta em cada fase do ciclo solar de Hale (22 anos). No inicio do ciclo, época de mínimo solar, a polaridade em um pólo é positiva (linhas de campo magnético saindo do Sol) e no outro é negativa (linhas de campo magnético entrando no Sol). Próximo ao máximo solar parece não existir polaridade dominante em nenhum pólo, e uma reversão da polaridade do Sol começa a tornar-se aparente cerca de 2 anos após o máximo solar (Kivelson e Russel, 1995; Hoyt e Schatten, 1997).

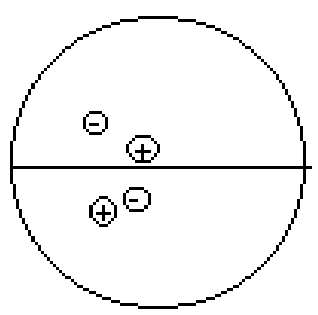

Figura 3. Diagrama indicando que pares de manchas solares ocorrem com polaridade oposta em ambos os hemisférios. A primeira mancha que surge em um par depende do hemisfério no qual as manchas se formam.

No mínimo solar, as manchas se formam a latitudes de cerca de $40^{\circ}$ em ambos os hemisférios solares. Raramente manchas solares aparecem em latitudes maiores do que $40^{\circ}$ e nunca no equador. À medida que o ciclo solar progride, o número de manchas primeiro aumenta e depois diminui em uma região larga que gradualmente se move para o equador. 
O início de um novo ciclo é caracterizado pelo reaparecimento de manchas em maiores latitudes. Este padrão de formação de manchas a cada ciclo em latitudes maiores e a migração para menores latitudes constitui um padrão denominado lei de Sporer, representado pelo diagrama de borboleta de Maunder, apresentado na Figura 4. O que está mudando é a localização das manchas sucessivas, enquanto as manchas individuais tipicamente duram apenas uma rotação solar ou menos e movem-se muito pouco relativamente a fotosfera.

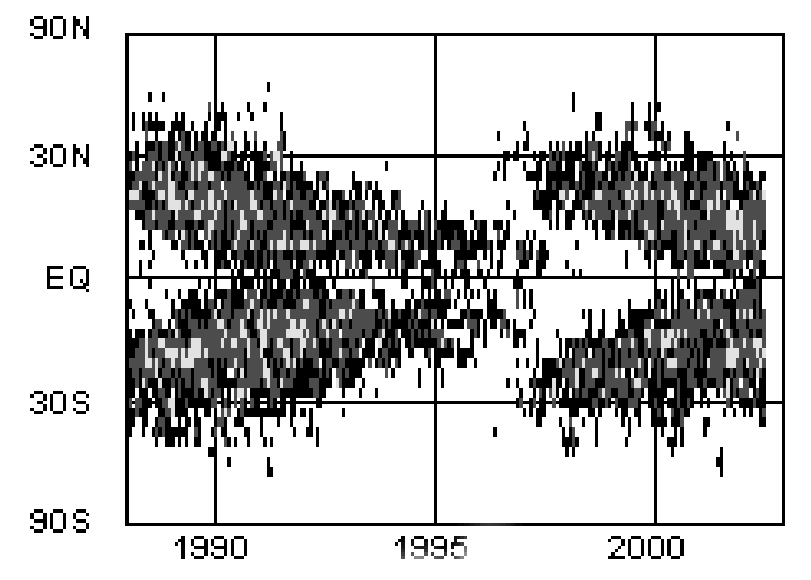

Figura 4. Diagrama de Maunder mostrando a localização (latitudes e longitudes heliográficas) da formação das manchas solares em função do tempo, em anos, para os ciclos solares mais recentes. Fonte: NASA, Sunsposts and Solar Cycle, http://www.sunspotcycle.com

A teoria proposta para explicar o movimento cíclico em latitude engloba produção e relaxação de tensão magnética por rotação diferencial. Por não ser um corpo sólido, o Sol não gira a uma velocidade uniforme, como a Terra, mas apresenta uma rotação menor em regiões equatoriais e maior em regiões polares. A rotação diferencial do Sol é em média entre 27-28 dias. Esta rotação diferencial produz maior tensão sobre o campo magnético próximo a $40^{\circ}$ de latitude. Devido à esta tensão, as linhas de campo magnético irrompem através da fotosfera, tornando-se o início para o desenvolvimento das manchas. Quando a tensão magnética, em torno de $40^{\circ}$, está sendo relaxada por erupções solares (solar flares), a tensão continua a crescer em menores latitudes solares à medida que o ciclo solar continua (Kivelson e Russel, 1995; Hoyt e Schatten, 1997).

A mancha solar é escura por ser mais fria do que a fotosfera vizinha. $\mathrm{O}$ esfriamento ocorre localmente, porque o campo magnético inibe a convecção de plasma das camadas sub-superficiais do Sol, mais quente que a superfície solar, e torna a temperatura da região menor. Por contraste com o restante da fotosfera, esta região mais fria será vista como uma região escura. Na fotosfera normal a convecção mistura as camadas superficiais com as sub-superficiais mais quentes e contribui para aquecer a superfície. Um tubo de fluxo magnético abaixo da superfície tende a subir pelo processo de empuxo magnético. O balanço de pressão lateral total (magnético e plasmático) entre o tubo de fluxo e sua região vizinha livre de campo implica que

$$
p+\frac{B^{2}}{2 \mu_{0}}=p_{0},
$$

em que $p$ é a pressão de plasma, $p_{0}$ é a pressão na vizinhança, $B^{2} / 2 \mu_{0}$ pressão magnética, e $\mu_{0}$ é a permissividade magnética. Esta equação implica que $p<p_{o}$, ou seja, a pressão de plasma dentro do tubo de fluxo é menor do que nas vizinhanças, e, se a diferença de temperatura não for muito grande, isto implicará que a densidade de plasma do tubo de fluxo será menor do que a das vizinhanças, e então o tubo de fluxo experimenta uma força de empuxo ascendente. Quando o tubo sobe e irrompe através da superfície solar, ele pode criar um par de manchas solares de polaridade oposta, como é freqüentemente observado.

Supõe-se que um mecanismo de dínamo opera através da zona de convecção, gerando o campo que oscila com um período de 11 anos. Um fator é a rotação diferencial do Sol, que estica um campo inicialmente poloidal e cria um campo toroidal. Um outro fator é que quando um elemento de fluxo toroidal sobe, ele tende a ser torcido por forças de Coriolis em um sentido que gera um novo fluxo poloidal de polaridade oposta.

\section{O Número de Manchas Solares}

Schwabe descobriu o ciclo de 11 anos em 1843 e Wolf nos anos de $1850 \mathrm{fez}$ um gigantesco esforço para compilar e reconstruir a atividade solar (Hoyt e Schatten, 1997). Ele teve sucesso em construir uma série de médias anuais desde 1700 (Eddy, 1976), o número de manchas solares de Wolf, também conhecido como número de Zurich ou Internacional, é definido como:

$$
R_{z}=k(10 g+f)
$$

Na equação $2, R_{z}$ é o número de manchas solares de Wolf, $k$ é uma constante de normalização para um observador particular, $g$ é o número de grupos de manchas solares e $f$ é o número de manchas solares individuais visíveis sobre o disco solar. A série de $R_{z}$ foi determinada por Wolf utilizando apenas um observador primário, e as observações que faltavam foram substituídas por observadores secundários. O fator de correção $k$ é usado para compensar diferenças sistemáticas em observações causadas por variações nos tamanhos de telescópio, condições atmosféricas e erros de observadores. A série temporal de $R_{z}$ não apresenta barras de erro, e muitas das antigas observações não foram usadas ou encontradas por Wolf em sua pesquisa. Os períodos sem dados foram preenchidos por Wolf com valores interpolados (Hoyt e Schatten, 1998). Para o período 1700-1748 somente médias anuais estão disponíveis, entre 1749-1817 médias mensais estão disponíveis e valores diários (porém com muitos intervalos sem dados, incluindo meses inteiros) estão disponíveis desde 1818. A Figura 5 apresenta as médias anuais de $R_{z}$ para o período entre 1700-2000. A oscilação característica do período de 11 anos é facilmente distinguida, assim como variações de períodos mais longos, com máximos do ciclo de 11 anos mais e menos intensos. Particularmente o período entre 1800-1830 que 
apresentou máximos menos intensos do ciclo solar, é conhecido como mínimo de Dalton da atividade solar. Dados de número de manchas solares podem ser obtidos a partir do National Geophysical Data Center, Boulder, Colorado, http://www.ngdc.noaa.gov/.

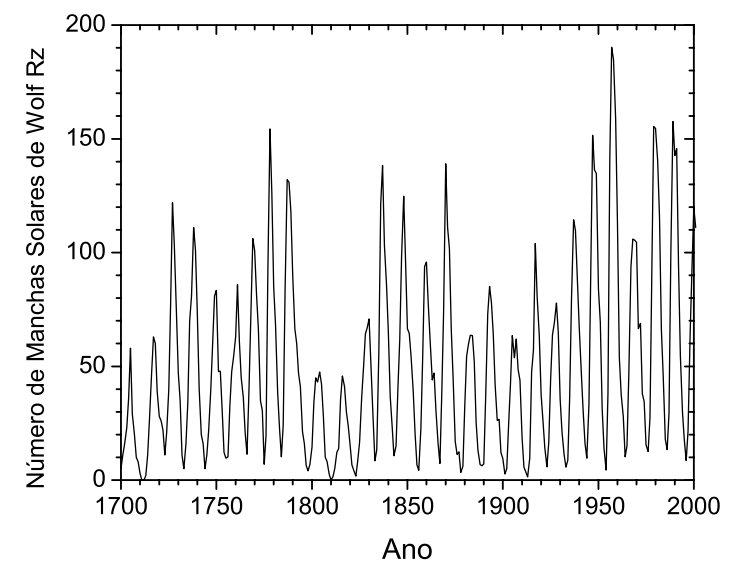

Figura 5. Série anual do Número de Manchas Solares de Wolf para o período 1700-2000.

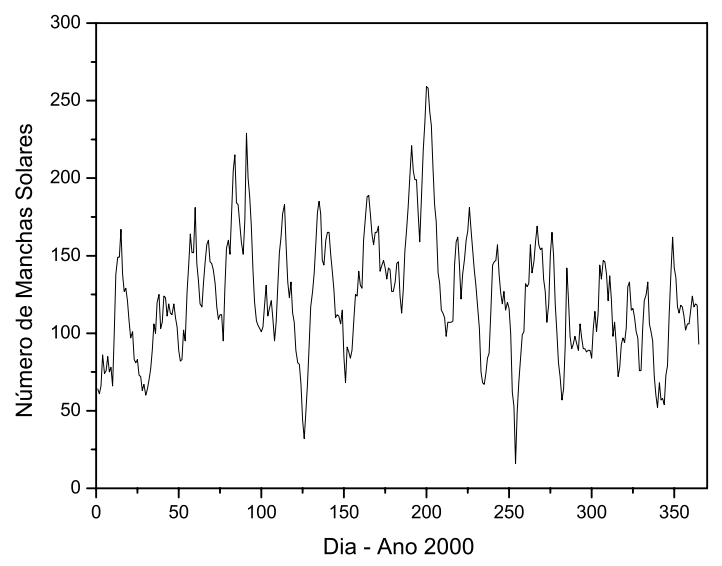

Figura 6. Valores diários de $R_{z}$ para o ano de 2000.

Os valores diários de $R_{z}$ variam muito mais do que as médias de longo período. No máximo solar os valores de $R_{z}$ podem ser maiores do que 100 por muitos dias, e no mínimo solar é comum observarem-se vários dias sem manchas. Na Figura 6 são apresentados os valores diários de $R_{z}$ para o ano de 2000.

A Figura 7 apresenta as médias mensais do período 1980 -2002, nota-se a variabilidade presente mês a mês. Também neste período, centrado no ciclo solar de 22 anos (máximo em 1990) pode-se observar a assimetria existente no ciclo solar. A fase ascedente, do mínimo para o máximo, é mais curta (média de 4 anos) do que a fase descendente, do máximo para o mínimo (5-6 anos). Este padrão repete-se nos ciclos solares em geral.

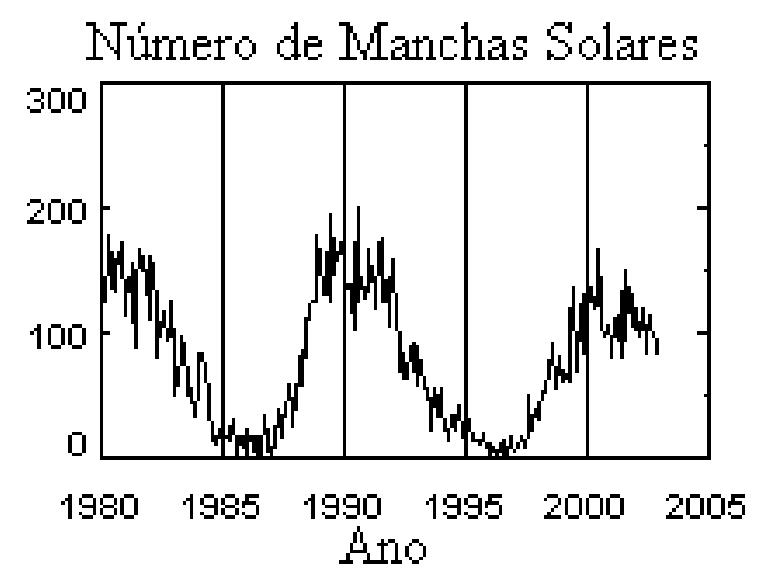

Figura 7. Ciclo solar apresentando a assimetria entre a fase ascendente, mais curta, e a fase descendente. FONTE: NASA, Solar Physics http://science.nasa.gov/ssl/pad/solar/sunspots.htm

As observações de manchas solares anteriores a 1850 são menos precisas do que as observações mais recentes (Hoyt e Shcatten, 1997). Uma nova análise das observações de manchas solares, realizada por Hoyt e Schatten (Hoyt e Schatten, 1997) e outros (Wilson, 1998), levou-os a concluir que a série do $R_{z}$ aparenta ter $25-50 \%$ a mais de amplitude, por volta de 1882, e que um novo parâmetro, o Número de Manchas Solares de Grupo, $R_{G}$, poderia ser um melhor indicador da atividade solar, especialmente para o período inicial (Hoyt e Schatten, 1998).

O número de manchas solares de Grupo $R_{G}$ foi construído por Hoyt e Schatten (1998) para ser mais autoconsistente (menos dependente de observações de manchas solares quase não visíveis) e menos ruidoso do que $R_{z}$. Observações diárias, mensais e anuais foram determinadas entre 1610-1995. Esta série foi determinada para ser similar ao $R_{z}$ :

$$
R_{G}=\frac{12.08}{N} \sum\left(k_{i}^{\prime} G_{i}\right),
$$

em que $G_{i}$ é o número de grupos de manchas solares observado pelo $i$-ésimo observador, $k_{i}^{\prime}$ é o fator de correção do $i$-ésimo observador, $N$ é o número de observadores utilizado para formar o valor diário e 12,08 é um número de normalização escolhido para fazer a média de $R_{G}$ idêntica a média de $R_{z}$ entre 1874-1976 (Hoyt e Schatten, 1998). A Figura 8 apresenta as médias anuais de $R_{G}$ no período 16101995. A série foi descontinuada após 1995, não tendo sido mais calculada (Hoyt e Schatten, 1998).

Nesta série é possível ver parte do mínimo de Maunder, período entre 1650-1715 em que a atividade solar medida pelo número de manchas solares foi muito baixa (Eddy, 1976) e inclusive em muitos anos não tendo sido constatada observações de manchas solares. Comparando as Figuras 5 e 8 observa-se que $R_{z}$ tem maiores amplitudes do que $R_{G}$ antes de 1882, sendo a amplitude das duas séries equivalente após 1882 (Hoyt e Schatten, 1998). Outra característica observada é de que no período de cerca de 1700-1750, a variabilidade de $R_{G}$ é maior do que àquela de $R_{z}$ (maior número de oscilações de alta frequiência). 


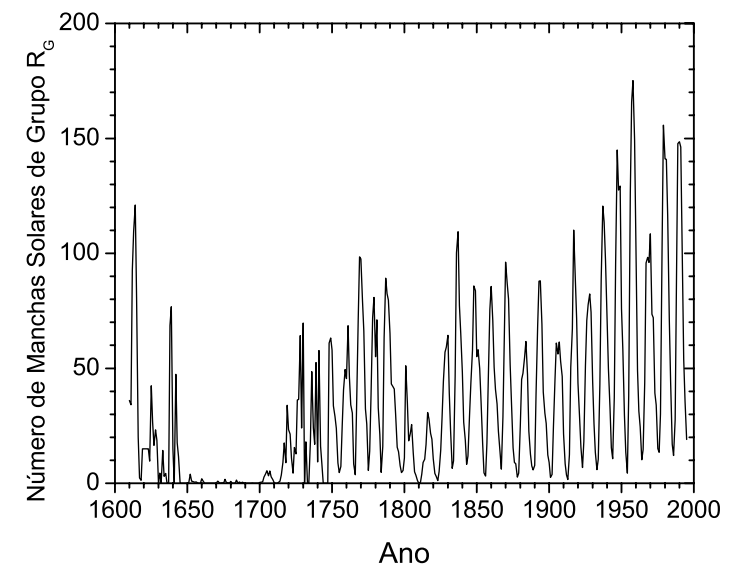

Figura 8. Série anual do número de manchas solares de Grupo para o período 1610-1995.

\section{Análise Espectral}

Comumente multiplica-se a série temporal por uma janela antes de realizar a transformada de Fourier para reduzir a vazamento espectral (potência da frequência real que vaza para freqüências vizinhas). Sem a janela, a parte de alta freqüência do espectro pode ser tendenciosa para o vazamento espectral (Parks et al., 1987). Entretanto, o uso de uma única janela em geral pondera desigualmente os pontos de dados e tende a aumentar a variância estatística da estimativa espectral. Sempre que apenas uma janela for utilizada, haverá um balanço entre a resistência ao vazamento espectral e a variância de uma estimativa espectral (Parks et al., 1987).

Thomson (1982) introduziu a técnica de análise espectral por janelamento múltiplo (Multiple Taper MethodMTM). Os dados são multiplicados não apenas por uma, mas por várias janelas resistentes ao vazamento, e cada janela amostra diferentemente a série. A informação estatística perdida pela primeira janela é recuperada pela segunda, e assim por diante. A estimativa de janelas múltiplas não é então restrita pelo balanço entre vazamento e variância como a estimativa por uma única janela. Este método utiliza janelas ortogonais para obter estimativas aproximadamente independentes do espectro de potência e então combiná-las em uma estimativa. Esta estimativa exibe mais graus de liberdade e permite uma quantificação mais fácil da tendência e do compromisso da variância, comparada à análise convencional de Fourier. O MTM tem a capacidade de detectar oscilações de pequena amplitude em uma série temporal curta sem a necessidade de filtrar o sinal. O método também possui um teste estatístico interno, teste-F (distribuição F), para obter o nível de significância das periodicidades encontradas (Thomson, 1982).

A Figura 9 apresenta o espectro de amplitude obtido com o MTM para a série do $R_{z}$. Os principais períodos aparecem identificados no gráfico. Ao nível de $95 \%$ de confiança, os períodos encontrados foram: 100,$7 ; 27,5 ; 21,6 ; 11,2 ; 4,6$; 3,$6 ; 3,4$ e 2,80 anos.

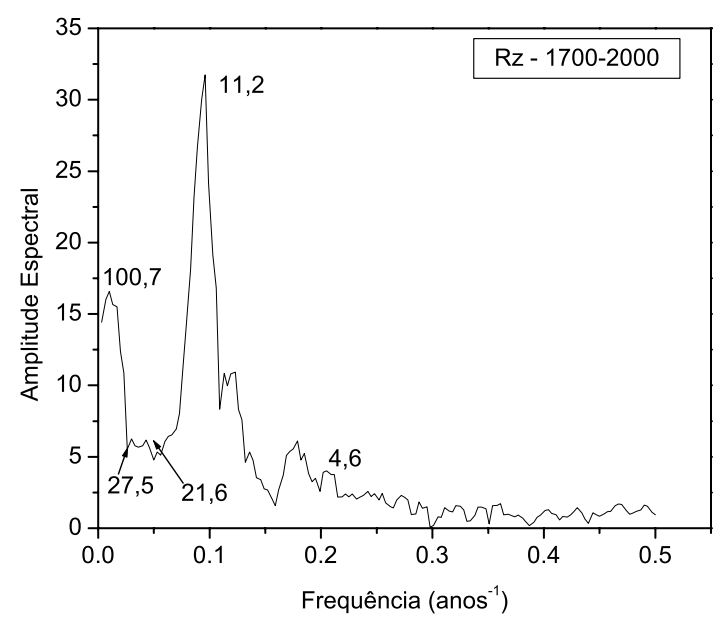

Figura 9. Espectro de amplitude do número de manchas solares de Wolf.

O principal pico no espectro visto na Figura 9 é claramente relacionado ao período do ciclo solar de 11 anos. Também é observado o período de 100,7 anos, associado ao ciclo de Gleissberg, o período de 21,6 anos, associado a ciclo magnético de 22 anos de Hale, e o período de 4,6 anos, provavelmente associado ao segundo harmônico do ciclo de Schwabe de 11 anos. O período de 27 anos e os períodos menores não tem uma associação clara a algum ciclo conhecido de variação da atividade solar.

Uma comparação do espectro usando o método de análise por regressão iterativa (Rigozo e Nordemann, 1998) mostrou que os components espectrais mais estatisticamente significativos, para o período 1700-1999, foram 65,$3 ; 52,0$; 12,$0 ; 11,0 ; 10,0$ e 8,5 anos (Rigozo et al., 2001). A análise espectral do número de Wolf também foi feita por vários autores. As características espectrais mais freqüentemente identificadas são: um conjunto de períodos relacionado ao sinal de 11 anos, períodos relacionados ao segundo harmônico solar do ciclo de 11 anos, ao ciclo magnético de Hale de 22 anos e ao ciclo de Gleissberg em torno de 80-100 anos (Herman e Goldberg, 1978). Alguns trabalhos recentes demonstraram resultados similares aos apresentados neste trabalho.

\section{Reconstrução do Número de Man- chas Solares}

Rigozo et al. (2001) reconstruíram o número de Wolf $R_{z}$ para um período de 1000 anos, utilizando a transformada ondeleta (wavelet) para decompor a série em níveis de frequiência e a análise por regressão iterativa para encontrar as principais periodicidades, amplitudes e fases. Utilizando a expressão: 


$$
R_{z}(t)=\sum_{1}^{n} r_{n} \sin \left(2 \pi \frac{t}{T_{n}}+\varphi_{n}\right)
$$

Eles calcularam a série para o período de 1000 a 2000 D.C., que se encontra reproduzida na Figura 10 juntamente com a identificação dos grandes máximos e mínimos da atividade solar. Dois grandes máximos na atividade solar são identificados, o Medieval (ao redor de 1150-1200 D.C.) e o Moderno (a partir de 1900) e 5 grandes mínimos, Oort (1090-1140 D.C.), Wolf (1300-1380 D.C.), Sporer (14101515 D.C.), Maunder (1641-1715 D.C.) e Dalton (17901825 D.C.). Estes períodos são coincidentes com máximos e mínimos da atividade solar identificados através de registros de radionuclídeos naturais, tais como o $\mathrm{C}^{14}$ (Damon e Lerman, 1978), mas foram obtidos de maneira totalmente independente, utilizando-se uma reconstrução matemática de periodicidades contidas nos níveis de frequiência de ondeleta da própria série do $R_{z}$.

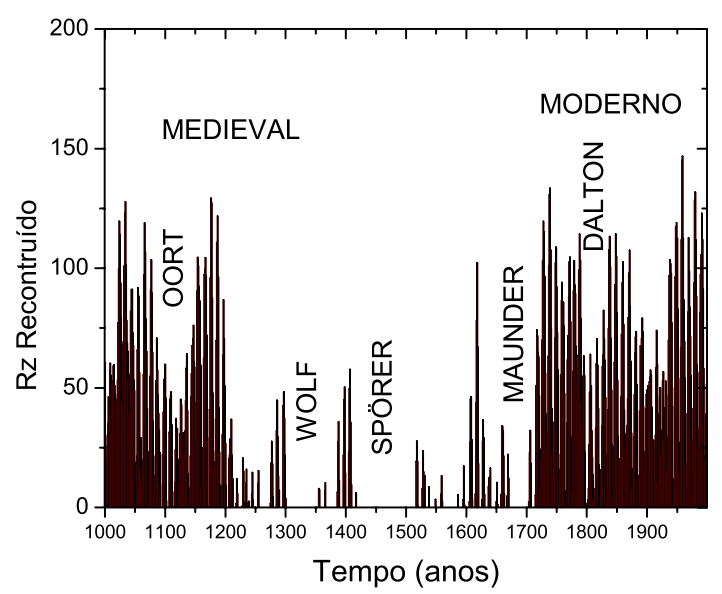

Figura 10. Reconstrução para 1000 anos do número de manchas solares de Wolf feita por Rigozo et al. (2001).

Pesquisas recentes têm demonstrado que os registros isotópicos em árvores apresentam informações significativas sobre as mudanças ambientais e as relações Sol-Terra no passado. Um monitoramento indireto dessas variações no passado foi feito por Stuiver e Quay (1980), através de medidas dos conteúdos do $\Delta^{14} \mathrm{C}$ produzidos na atmosfera terrestre e assimilados pelas árvores. Mudanças na razão de produção do ${ }^{14} \mathrm{C}$ estão associadas as magnitudes da atividade solar, que apresentam uma relação inversa - nas épocas que a atividade solar é máxima existe uma baixa produção do ${ }^{14} \mathrm{C}$ e nas épocas que a atividade solar é mínima existe uma maior produção do ${ }^{14} \mathrm{C}$-, estas flutuações se apresentam em escalas seculares bem como em escalas decadal, incluindo o ciclo solar de 11 anos. A existência de tais variações seculares causadas pela atividade solar também tem sido confirmada pelas concentrações do ${ }^{10} \mathrm{Be}$ no gelo (Beer et al., 1988). Através desta relação inversa entre os radionuclídeos cosmogênicos e a atividade solar, acredita-se que nos últimos 1000 anos o Sol apresentou algumas épocas em que estava bastante ativo e outras em que sua atividade era baixa. Estas épocas são chamadas de grandes máximos e mínimos da atividade solar.

A relação inversa entre raios cósmicos e atividade solar é explicada porque os radionuclídeos são o produto da reação de raios cósmicos galácticos (RCG) com constituintes da alta atmosfera. $\mathrm{Na}$ atividade solar máxima, o campo magnético heliosférico é mais irregular e intenso, refletindo mais os RCG, reduzindo sua intensidade na terra, e, portanto, a produção de radionuclídeos é menor. Durante períodos de menor atividade solar, o campo magnético heliosférico, mais fraco, desvia menos os RCG e estes chegam em maior intensidade na alta atmosfera da Terra, aumentando a produção de radionuclídeos.

A Figura 11 apresenta uma superposição de dados medidos do $\mathrm{C}^{14}$ para o último milênio com a Figura 10 (reconstrução das manchas solares para o último milênio). Os mínimos de Dalton e Oort foram mínimos menos intensos do que os de Wolf, Sporer e Maunder, onde a atividade solar diminui, mas não tanto (Hoyt e Schatten, 1997). O máximo medieval e o mínimo de Maunder, particularmente, são coincidentes com períodos de anomalias climáticas, particularmente temperaturas mais altas (no máximo Moderno) e mais frias (no mínimo de Maunder) no hemisfério norte. O que pode ser interpretado como indicador de uma possível influência da atividade solar sobre o clima terrestre (Hoyt e Schatten, 1997).

Uma questão interessante foi levantada por Rigozo et al. (2001). O nível de $R_{z}$ no mínimo de Oort corresponde ao nível no mínimo de Dalton, e ambos foram seguidos por máximos (Medieval e Moderno). Seria possível então que este padrão seja reproduzido no futuro, isto é, o presente Máximo Medieval poderá ser seguido por três mínimos pronunciados, tais como os de Wolf, Sporer e Maunder? E o clima da Terra poderia ser afetado de maneira similar ao passado, com a observação de uma tendência de resfriamento?

Embora na atualidade se observe uma tendência no aquecimento global do clima, atribuída principalmente ao efeito estufa aumentado devido a maiores concentrações atmosféricas de $\mathrm{CO}_{2}$, nem toda variação da temperatura global pode ser atribuída a este gás, e o papel da atividade solar no clima da Terra, como e quanto o influencia, vem sendo amplamente pesquisado e debatido na comunidade científica (Hoyt e Schatten, 1997). Assim, não se pode descartar a possibilidade de que, apesar do $\mathrm{CO}_{2}$ continuar aumentando suas concentrações, uma redução da atividade solar nas próximas décadas/séculos pode contribuir para o resfriamento do clima. 


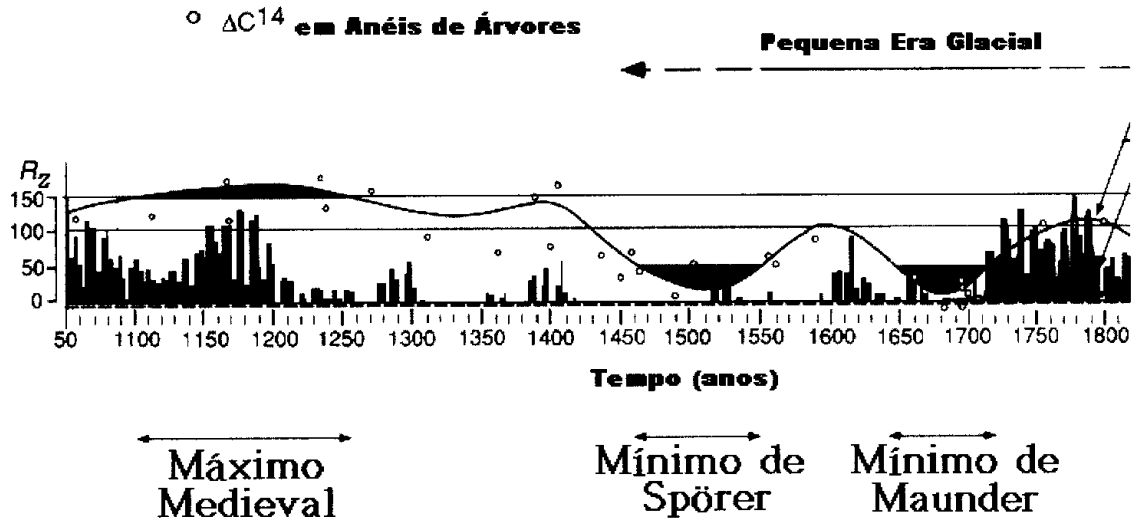

Figura 11. Comparação entre a série de ${ }^{14} \mathrm{C}$ de Stuiver e Quay (1980) e o número de Manchas Solares de Wolf calculado por Rigozo et al. (2001).

\section{Conclusões}

Este trabalho apresentou uma breve revisão sobre os conceitos básicos referentes às manchas solares, características escuras na superfície solar por possuírem intensos campos magnéticos que bloqueiam a convecção sub-fotosférica e as tornam mais frias do que a superfície solar. Apresentaramse o número de manchas solares de Wolf e de Grupo, e comentou-se sobre o ciclo solar de 11 anos e outros períodos. A reconstrução para 1000 anos realizada por Rigozo et al (2001) também foi apresentada com a correspondente série de radionuclídeo $\mathrm{C}^{14}$ apresentando boa concordância. O número de manchas solares é um indicador geral da atividade magnética do Sol, e inúmeros fenômenos solares e interplanetários, que afetam diretamente o ambiente eletromagnético terrestre (magnetosfera), têm variação modulada por este ciclo, tais como a ocorrência de ejeções coronais de massa, de ondas de choque interplanetário: explosões solares e eventos de prótons solares. Em consequiência, a ocorrência de tempestades geomagnéticas e outras perturbações na magnetosfera terrestre é maior durante o máximo do ciclo solar. Também a incidência de raios cósmicos galácticos na Terra é observada ser anticorrelacionada ao ciclo das manchas solares, pois durante períodos de máximo solar o campo magnético na heliosfera tem uma estrutura mais complexa, e blinda melhor o Sistema Solar interno (Kivelson e Russel, 1995). Variações com o ciclo solar na alta atmosfera da Terra (ionosfera) e na camada de ozônio terrestre são bem conhecidas. Possíveis efeitos do ciclo solar no clima da Terra têm sido estudados e embora este assunto ainda seja controverso, há várias evidências indicando uma variação de 11 anos em vários parâmetros climáticos (Hoyt e Schatten, 1997).

\section{Referências}

[1] P. E. Damon, J. C. Lerman e A. Long, Annual Review of Earth Planetary Science, 6, 457 (1978).

[2] J. A. Eddy, Science, 192, 1189 (1976).

[3] J. R. Herman e R. Goldberg, Sun, Weather and Climate, Scientific and Technical Information Branch, National Aeronautics and Space Administration, Washington, D.C. (1978).

[4] D. V. Hoyt e K. H. Schatten, The role of the Sun in Climate Change, Oxford University Press, New York (1997).

[5] D. V. Hoyt. e K. H. Schatten, Solar Phys., 179, 189 (1998).

[6] M.G. Kivelson, M. G. e C. T. Russell, Introduction To Space Physics, Cambridge University Press, Cambridge (1995).

[7] R. B. Leighton, Astrophysic Journal, 156, 1 (1969).

[8] J. Parks, C. R. Lindberg, C. R. e D. J. Thomson, Geophysical Journal Royal Astronomical Society, 91, 755 (1987).

[9] N. R. Rigozo, E. Echer, L. E. A. Vieira D. J. R. Nordemann, D. J. R., Solar Physics, 203, 179 (2001).

[10] N. R. Rigozo D. J. R. Nordemann, Rev. Bras. Geofís. 16, 151 (1998).

[11] D. J. Schove, Sunspot Cycles, Hutchinson Ross Publishing Company, Stroudsburg, Pennsylvania (1983).

[12] M. Stuiver e P. D. Quay, Science, 207, 11 (1980).

[13] D. J. Thomson, Proc. IEEE, 70, (1055) 1982.

[14] R. M. Wilson, Solar Phys., 182, 217 (1998). 